\title{
BIOESTIMULANTE EN EL CRECIMIENTO Y CALIDAD DE PLÁNTULAS DE PLÁTANO EN FASE DE VIVERO
}

\section{BIOSTIMULANT IN THE GROWTH AND QUALITY OF BANANA SEEDLINGS IN THE NURSERY PHASE}

\author{
Galo Alexander Cedeño García ${ }^{1}$, Sofía del Rocío Velásquez Cedeño ${ }^{1}$, Benny Alexander Avellán Cedeño ${ }^{2}$, Jessica Elizabeth \\ Cargua Chávez ${ }^{3}$, Geoconda Aracely López Álava ${ }^{1}$
}

${ }^{1}$ Escuela Superior Politécnica Agropecuaria de Manabí Manuel Félix López, Campus Politécnico El Limón, km 2 1⁄2 vía Calceta - El Gramal. Calceta, Manabí, Ecuador.

${ }^{2}$ Instituto Nacional de Investigaciones Agropecuarias (INIAP), Estación Experimental Portoviejo, km 12 vía Portoviejo-

Santa Ana, Portoviejo, Manabí, Ecuador

${ }^{3}$ Instituto Superior Tecnológico Tsa'chila, Av. Galo Luzuriaga y Calle B, Santo Domingo de los Tsáchilas, Ecuador.

Email: gcedeno@espam.edu.ec

\section{Información del artículo}

Tipo de artículo: Artículo original

Recibido:

07/09/2021

Aceptado:

27/12/2021

Licencia:

CC BY-NC-SA 4.0

Revista

ESPAMCIENCIA

12(2):124-130

DOI:

https://doi.org/10.5 1260/revista_espam ciencia.v12i2.274

\section{Resumen}

El objetivo de la investigación fue evaluar la efectividad de un bioestimulante de extracto de algas marinas sobre el crecimiento y calidad de plántulas de plátano en etapa de vivero. Los tratamientos evaluados fueron: bioestimulante + fertilización química (T1), fertilización química (T2) y control (T3). Se utilizó un diseño completamente aleatorizado con tres tratamientos y siete repeticiones. Se utilizaron rizomas de $200 \mathrm{~g}$ de peso, que fueron sembrados en contenedores de polietileno, donde se dejaron emerger y crecer hasta completar 20 $\mathrm{cm}$ de altura. Seguidamente se aplicó los tratamientos y se registró la masa seca inicial de las plántulas con la finalidad de medir el incremento de crecimiento por efecto de los tratamientos. A los 60 días después de primera aplicación de tratamientos se registró el área foliar, masa seca e índice de calidad de Dickson. Los datos se sometieron a análisis de varianza y la separación de medias con prueba de Tukey $(\alpha<0,05)$. Además, se realizó análisis de correlación entre las variables de crecimiento y de calidad de las plántulas. El bioestimulante + fertilización química, incrementó el área foliar, masa seca e índice de calidad de Dickson de las plántulas en $14,80,11,22$ y 7,56\%, respectivamente, con relación al tratamiento de fertilización química. El índice de calidad de Dickson correlacionó positivamente con el crecimiento de las plántulas. El uso de bioestimulante de extracto de algas marinas fue efectivo para mejorar el crecimiento y calidad de las plántulas de plátano en vivero, y potenció el efecto de la fertilización edáfica.

Palabras clave: Musa AAB, biorreguladores, plantas, aclimatación, desarrollo, vigor

\section{Abstract}

The objective of the research was to evaluate the effectiveness of a marine algae extract biostimulant on the growth and quality of plantain seedlings in the nursery stage. The evaluated treatments were: Biostimulant + chemical fertilization (T1), Chemical fertilization (T2) and Control (T3). A completely randomized design with three treatments and seven repetitions was used. Rhizomes weighing $200 \mathrm{~g}$ were used, which were sown in polyethylene containers, where they were allowed to emerge and grow until reaching $20 \mathrm{~cm}$ in height. Then the treatments were applied and the initial dry mass of the seedlings was recorded in order to measure the increase in growth due to the effect of the treatments. At 60 days after the first application of treatments, the foliar area, dry mass and Dickson's quality index were recorded. The data were subjected to analysis of variance and separation of means with Tukey's test $(\alpha<0.05)$. In addition, correlation analysis was performed between the growth and quality variables of the seedlings. The biostimulant + chemical fertilization increased the leaf area, dry mass and Dickson's quality index of the seedlings by $14.80,11.22$ and $7.56 \%$, respectively, in relation to the chemical fertilization treatment. Dickson's quality index positively correlated with seedling growth. The use of biostimulant from seaweed extract was effective to improve the growth and quality of plantain seedlings in the nursery, and enhanced the effect of edaphic fertilization.

Keywords: Musa AAB, bioregulators, plants, acclimatization, development, vigor 


\section{INTRODUCCIÓN}

El plátano (Musa AAB Simmonds) tiene importancia en el orden alimentario, social y económico a nivel local y global, debido a que contribuye a la generación de divisas, fuentes de empleo y sustenta la seguridad alimentaria de gran parte de la población, dado su alto valor energético y nutritivo (Sepúlveda et al., 2017; Falcomer et al., 2019; Scott, 2021). La principal problemática es la baja productividad, influenciada por factores limitantes como: déficit hídrico por una prolongada época seca, falta de riego complementario, pues el $81 \%$ de la superficie se cultiva bajo secano, uso de semillas de baja calidad y material genético tradicional, plantaciones en avanzada edad y densidades de siembra inadecuadas (Zambrano et al., 2018; INEC, 2020; Cedeño et al., 2020).

En este contexto, renovar e incrementar el área de siembra con material genético de calidad es imprescindible para aumentar el potencial productivo, más aún, cuando en siembras tradicionales, no se considera la calidad de las plántulas como factor determinante en la capacidad adaptativa y sobrevivencia al estrés pos trasplante, y por ende en la expresión del potencial productivo (Staver y Lescot, 2015; Jacobsen et al. 2019; Galán et al., 2018). La calidad de una planta se relaciona en gran medida con el vigor, la supervivencia y el crecimiento de las plantas después del trasplante (Grossnickle y MacDonald, 2018; Lin et al., 2019). Previo al trasplante a campo definitivo, las plántulas necesitan una fase previa de enraizamiento, aclimatación o endurecimiento en vivero, puesto que sin esta etapa muestran altas tasas de mortalidad y son incapaces de adaptarse y sobrevivir a condiciones de campo, más aún si provienen de propagación in vitro (Galán et al., 2018; Parkhe et al., 2019; El-Mahrouk y ElShereif, 2019).

Actualmente, el uso de bioestimulantes en etapas previas al trasplante es propuesto como estrategia para fortalecer el crecimiento de las plántulas, su capacidad adaptativa al estrés y supervivencia en campo (Qin y Leskovar, 2020; Malik et al., 2021; Reyes et al., 2021). De acuerdo a consensos científicos, los bioestimulantes tanto microbianos y no microbianos son capaces de inducir respuestas morfoanatómicas, bioquímicas, fisiológicas y moleculares de las plantas, el aumento de la productividad de los cultivos, la eficiencia de uso de nutrientes y el aumento de la tolerancia contra el estrés (Rouphael y Colla, 2020). A juicio de los expertos, en investigaciones recientes, el efecto positivo de bioestimulantes microbianos y no microbianos sobre el crecimiento de plántulas de banano y plátano en fase de aclimatación en vivero (Izquierdo et al., 2012; Mateus y Rodríguez, 2019; Mora et al., 2021). Sin embargo, a nivel local, no se evidencia si los bioestimulantes aplicados en etapa de vivero mejoren la calidad de plántulas de plátano.. Por lo anterior expuesto, este trabajo tuvo como objetivo evaluar la efectividad de un bioestimulante sobre el crecimiento y calidad de plántulas de plátano en etapa de vivero.

\section{MATERIALES Y MÉTODOS}

\section{Localización}

El trabajo se desarrolló desde junio a diciembre de 2020 en el vivero de la Escuela Superior Politécnica Agropecuaria de Manabí Manuel Félix López, en Calceta, Manabí, Ecuador, ubicado en n las coordenadas $0^{\circ} 49^{\prime} 10^{\prime \prime}$ de latitud sur y $80^{\circ} 10^{\prime} 40^{\prime \prime}$ de longitud oeste, a una altitud de 21 m.s.n.m., con temperatura media anual de $25,7^{\circ} \mathrm{C}$, precipitaciones anuales promedio de $839 \mathrm{~mm}$ y heliofanía de 1045 horas anuales.

\section{Material vegetal}

Se utilizaron cebollines de plátano cv. Barraganete de 200 $\mathrm{g}$, los cuales fueron colocados en contenedores de polietileno de $15 \times 22 \mathrm{~cm}$, llenas previamente con sustrato compuesto de cascarilla de arroz, arena de río y vermicompost en relación volumétrica 1:1:1. Previo a la siembra los cebollines se limpiaron con ayuda de un cuchillo hasta dejarlos completamente blancos, con la finalidad de remover restos biológicos de insectos, nematodos y patógenos. Posteriormente, para mejorar su estado sanitario y de acuerdo con las recomendaciones de Coyne et al. (2010), fueron sumergidos en agua hirviendo durante 25 minutos,

Una vez que los cebollines fueron sembrados en los contenedores, se dejaron emerger y crecer hasta que completaran una altura de $20 \mathrm{~cm}$ por encima del nivel del sustrato, momento en el cual se procedió al registro de la masa seca de plántulas con ayuda de estufa y balanza analítica, con la intención de medir el ritmo de crecimiento diario a los 15, 30, 45 y 60 días después de la aplicación de tratamientos.

\section{Tratamientos}

Los tratamientos evaluados fueron:

$\mathbf{T}_{1}$ : Bioestimulante + fertilización química

$\mathbf{T}_{2}$ : Fertilización química

$\mathbf{T}_{3}$ : Control

\section{Diseño y unidad experimental}

Se utilizó un diseño completamente aleatorizado (DCA) con tres tratamientos, siete repeticiones y 21 unidades experimentales. La unidad experimental se conformó por 10 plántulas. 


\section{Aplicación de tratamientos}

Para el tratamiento con bioestimulación, se utilizó un producto comercial compuesto por Extracto de algas marinas noruegas (Ascophyllum nodosum), macro y micronutrientes, reguladores de crecimiento (auxinas, giberelinas, citocininas), carbohidratos, proteínas, ácidos orgánicos, aminoácidos y vitaminas. Este producto se aplicó con una concentración de $5 \mathrm{~mL} \cdot \mathrm{L}^{-1}$ de agua, y de esta solución se aplicó en drench una dosis de $25 \mathrm{~mL}$ planta $^{-1}$, a los $0,15,30$ y 45 días después de iniciado el experimento. La aplicación se realizó con bomba manual de presión con dosificador.

Para el tratamiento con fertilización química se utilizó un fertilizante compuesto de $15 \%$ de N, $15 \%$ de $\mathrm{P}_{2} \mathrm{O}_{5}$ y $15 \%$ de $\mathrm{K}_{2} \mathrm{O}$, que fue aplicado alrededor de la plántula en dosis de 10 g.planta ${ }^{-1}$, a los 15 días de iniciado el experimento. Las plántulas tratadas con bioestimulante también recibieron esta misma dosis de fertilización edáfica.

\section{Registro de variables}

Los datos registrados fueron variables morfométricas como altura de plántula, diámetro de tallo y longitud de biomasa radical a los 60 días de iniciado los tratamientos. La altura se midió en $\mathrm{cm}$ desde el nivel del sustrato hasta la $\mathrm{V}$ formada por las dos últimas hojas. El diámetro de tallo se registró en mm con la ayuda de un calibrador a nivel del sustrato. La longitud de biomasa radical fue medida en $\mathrm{cm}$ desde la base del rizoma hasta el ápice del conjunto de raíces. El área foliar fue estimada con la ecuación [1]. El índice de calidad de Dickson (ICD) fue estimado con la ecuación [2].

La masa seca de plantas fue registrada en $\mathrm{g}$ a los 15,30 , 45 y 60 días de crecimiento, para lo cual fueron fragmentadas en pedazos pequeños y secados en estufa de ventilación forzada a $75^{\circ} \mathrm{C}$ hasta alcanzar peso constante. Con los datos de masa seca se calculó el ritmo de crecimiento diario, para lo cual se utilizó la ecuación [3].

Área Foliar $\left(\mathrm{cm}^{2}\right)=L H * A H * K(0,80) * N H *$ $K_{2}(0,662)$

Donde:

$\mathrm{LH}=$ longitud de tercera hoja, $\mathrm{AH}=$ ancho de tercera hoja, $\mathrm{K}=$ factor de curvatura de Murray (1960), $\mathrm{NH}=$ número de hojas y $\mathrm{K}_{2}=$ nuevo factor de curvatura de Kumar et al. (2002).

$$
\begin{gathered}
I C D=\frac{\text { Masa seca biomasa total }(g)}{\frac{\text { Altura de planta }(\mathrm{cm})}{\text { Diámetro de tallo }(\mathrm{mm})}+\frac{\text { Masa seca aéreo }(g)}{\text { Masa seca radical }(g)}} \\
\mathrm{RCD}=\left[\left(\mathrm{M}_{\mathrm{F}}-\mathrm{M}_{\mathrm{I}}\right) /\left(\mathrm{T}_{\mathrm{F}}-\mathrm{T}_{\mathrm{I}}\right)\right]
\end{gathered}
$$

Donde:

$\mathrm{RCD}=$ ritmo de crecimiento diario, $\mathrm{M}_{\mathrm{F}}=$ masa seca final, $\mathrm{M}_{\mathrm{I}}=$ masa seca inicial, $\mathrm{T}_{\mathrm{F}}=$ tiempo final, $\mathrm{T}_{\mathrm{I}}=$ tiempo inicial

\section{Análisis de datos}

Los datos fueron sometidos a análisis de varianza al 5\% de probabilidades de error, la separación de medias con prueba de Tukey al 5\% de probabilidades de error. Además, se realizaron correlaciones entre variables de crecimiento y calidad, y regresión del ritmo de crecimiento de masa seca.

\section{RESULTADOS Y DISCUSIÓN}

Los tratamientos evaluados influyeron significativamente $(\mathrm{p}<0.05)$ sobre el crecimiento morfométrico de las plántulas de plátano (Cuadro 1). El tratamiento con bioestimulante + fertilización química incrementó la altura de planta en 8,04 y $24,62 \%$, en relación a los tratamientos con fertilización química y el control, respectivamente. El diámetro de tallo fue incrementado en 8,77 y $25,05 \%$ por el tratamiento bioestimulante + fertilización química, en relación a la fertilización química y el tratamiento control, respectivamente. De la misma forma, el tratamiento con bioestimulante + fertilización química alcanzó la mayor longitud de biomasa radical, con un incremento del 12,82 y $23,70 \%$, con respecto a los tratamientos con fertilización química y control, en su orden respectivo (Cuadro 1).

Resultados similares reportaron Moya et al. (2016) quienes han verificado que en la fase de aclimatación existe mayor diámetro de tallo y longitud radical en plántulas de banano tratadas con ácidos húmicos y vermicompost, en relación a plántulas no tratadas. Al respecto, Ewane et al. (2019) mostraron incrementos del 32 y $30 \%$ en altura y diámetro de tallo en plántulas de plátano que recibieron aplicación de bioestimulante en vivero, en comparación al tratamiento control. Teniendo en cuenta a Mora et al. (2021) en plántulas de banano tratadas con biofertilizante y microorganismos durante fase de vivero, en comparación al tratamiento control se obtuvo incrementos de 14,5; 19,3 y 91,8\% de altura de planta, diámetro de tallo y longitud radical, respetivamente. De manera similar, Martínez et al. (2021) alcanzaron incrementos de $10 \mathrm{~cm}$ y $19 \mathrm{~g}$ en altura de planta y peso seco de raíces, en plántulas de banano tratadas en vivero con vermicompost líquido y sólido, con relación a las plántulas no tratadas. 
Cuadro 1. Efecto de bioestimulante sobre variables morfométricas de plántulas de plátano a los 60 días de aclimatación en vivero.

\begin{tabular}{cccc}
\hline Tratamientos & $\begin{array}{c}\text { Altura de } \\
\text { planta }(\mathrm{cm})\end{array}$ & $\begin{array}{c}\text { Diámetro de } \\
\text { tallo }(\mathrm{mm})\end{array}$ & $\begin{array}{c}\text { Longitud de } \\
\text { biomasa } \\
\text { radical }(\mathrm{cm})\end{array}$ \\
\hline $\begin{array}{c}\text { Bioestimulante }+ \\
\text { fertilización química }\end{array}$ & $40,57 \mathrm{a}^{1 /}$ & $38,08 \mathrm{a}$ & $37,04 \mathrm{a}$ \\
Fertilización química & $37,31 \mathrm{~b}$ & $34,74 \mathrm{~b}$ & $32,29 \mathrm{~b}$ \\
Control & $30,58 \mathrm{c}$ & $28,54 \mathrm{c}$ & $28,26 \mathrm{c}$ \\
\hline p-valor ANOVA & $<0,0001$ & $<0,0001$ & $<0,0001$ \\
C.V. $\%$ & 3,86 & 3,82 & 5,94 \\
\hline 1/Medias dentro de columnas con & letras distintas, difieren \\
estadísticamente de acuerdo a la prueba de Tukey $\alpha<0.05$ &
\end{tabular}

La combinación de bioestimulante + fertilización química también influenció significativamente $(\mathrm{p}<0,05)$ la acumulación de masa seca, el área foliar e índice de calidad de Dickson, con relación a los tratamientos de fertilización química y control (Cuadro 2). El bioestimulante + fertilización química promovió mayor acumulación de masa seca, con incrementos del 11,22 y $23,98 \%$ con respecto a la fertilización química y tratamiento control. Del mismo modo, las plántulas que recibieron el bioestimulante + fertilización química alcanzaron mayor área foliar, con incrementos del 14.81 y $30,47 \%$, en comparación a los tratamientos con fertilización química y control, respectivamente. La calidad de las plántulas fue superior con el tratamiento del bioestimulante + fertilización química en 7,56 y 20,08\%, en relación a los tratamientos de fertilización química y control en su orden respectivo (Cuadro 2).

Cuadro 2. Efecto de bioestimulante sobre el crecimiento y calidad de plántulas de plátano a los 60 días de aclimatación en vivero.

\begin{tabular}{cccc}
\hline Tratamientos & $\begin{array}{c}\text { Masa seca } \\
\text { de planta }(\mathrm{g})\end{array}$ & $\begin{array}{c}\text { Área foliar } \\
\left(\mathrm{cm}^{2}\right)\end{array}$ & $\begin{array}{c}\text { Índice de } \\
\text { calidad de } \\
\text { Dickson (ICD) }\end{array}$ \\
\hline $\begin{array}{c}\text { Bioestimulante }+ \\
\text { fertilización química }\end{array}$ & $57,04 \mathrm{a}$ & $3230,66 \mathrm{a}$ & $13,20 \mathrm{a}$ \\
Fertilización química & $50,64 \mathrm{~b}$ & $2752,21 \mathrm{~b}$ & $12,19 \mathrm{~b}$ \\
Control & $43,36 \mathrm{c}$ & $2246,40 \mathrm{c}$ & $10,55 \mathrm{c}$ \\
\hline p-valor ANOVA & $<0,0001$ & $<0,0001$ & $<0,0001$ \\
C.V. \% & 7,38 & 4,16 & 3,16 \\
\hline
\end{tabular}

1/Medias dentro de columnas con letras distintas, difieren estadísticamente de acuerdo a la prueba de Tukey $\alpha<0.05$

Los resultados se asemejan a los informados por Izquierdo et al. (2012), quienes reportaron una mayor acumulación de masa seca y área foliar en plántulas in vitro de banano que recibieron aplicación de un análogo espirostánico de brasinoesteroides en fase de aclimatación. Asimismo, Mukhongo et al. (2015) alcanzaron incrementos de masa seca de plántulas de banano del 34, 46 y 33\%, respectivamente, con aplicación de tres combinaciones de bioestimulantes de origen microbiano, en relación al tratamiento control. También, los resultados obtenidos por
Mateus y Rodríguez (2019) que reportaron mayor acumulación de masa seca y tasa fotosintética en plántulas de plátano tratadas con bioestimulantes. En este mismo contexto, Mora et al. (2021) mostraron incrementos del 13,4 y $90,5 \%$ de área foliar y peso seco en plántulas de banano tratadas con biofertilizante y microorganismos durante fase de vivero, en comparación al tratamiento control. Los resultados de índice de calidad de Dickson, fueron próximos a los obtenidos por Rodríguez y Ramírez (2006) en plántulas de banano en fase de vivero, quienes reportaron promedios de hasta 12 puntos de ICD.

El ritmo de crecimiento diario basado en acumulación de masa seca varió significativamente $(p<0,05)$ con los tratamientos evaluados (Figura 1). En todos los tratamientos se observó una tendencia cuadrática, donde a partir de los 45 días el ritmo de crecimiento empieza a ralentizarse, posiblemente debido a que el volumen del contenedor comienza a ser limitante para el crecimiento radical de las plántulas. Se observó mayor incremento del ritmo de crecimiento con el tratamiento bioestimulante + fertilización química, donde entre los 0-15 días superó a la fertilización química y control con el 23,91 y 41,30\%, respectivamente. Entre los 16-30 días el bioestimulante + fertilización química incrementó el ritmo de crecimiento en 20 y $40 \%$, con respecto a los tratamientos de fertilización química y control. Así mismo, el bioestimulante + fertilización química incrementó el ritmo de crecimiento en 10,77 y 35,38\%, con relación a la fertilización química y tratamiento control entre los 31-45 días de crecimiento en vivero. Finalmente, entre los 46-60 días de crecimiento, el bioestimulante + fertilización química mostró un incremento mayor a los tratamientos de fertilización química y control, con 8,93 y 32,14\%, en su respectivo orden (Figura 1).

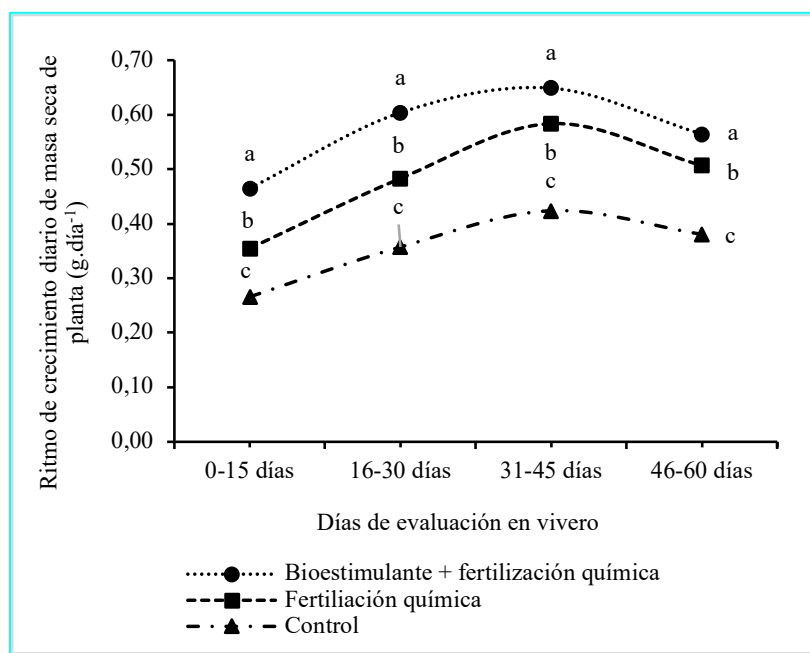

Figura 1. Ritmo de crecimiento diario a base de masa seca de plántulas de plátano en fase de vivero. Tratamientos con letra distinta en los periodos de crecimiento evaluados difieren estadísticamente según la prueba de Tukey $\alpha<0.05$. 
En este sentido, los resultados de acumulación de masa seca se asemejan a los reportados por Izquierdo et al. (2012), Mukhongo et al. (2015), Mateus y Rodríguez (2019) y Mora et al. (2021) que consiguieron incrementar la masa seca de plántulas de banano en aclimatación con aplicación de diversos bioestimulantes. Los resultados obtenidos también evidencian que los bioestimulantes mejoran el uso de nutrientes y potencian el efecto de la fertilización, dado que las plántulas que recibieron aplicación de bioestimulante, también recibieron fertilización química edáfica, lo cual se reflejó en mayor crecimiento y calidad de las plántulas (Cuadro 1 y 2).

En este contexto, el efecto de los bioestimulantes sobre el crecimiento de plántulas en vivero se debe a su diversa y compleja composición química de sustancias bioactivas como hidrolizados de proteínas, extractos de algas, ácidos fúlvicos, ácidos húmicos, compuestos nitrogenados, bacterias beneficiosas y agentes fúngicos, que estimulan la fisiología y la bioquímica de las plantas, como la proporción de pigmentos fotosintéticos de las hojas (carotenoides y clorofila), un mayor potencial antioxidante, un enorme crecimiento de las raíces, una mayor eficiencia en el uso de nutrientes (EUN) y una mayor capacidad de tolerar el estrés pos trasplante (Colla et al., 2015; Qin y Leskovar, 2020; Rouphael y Colla, 2020; Malik et al., 2021; Ali et al., 2021).

Independientemente del efecto de los tratamientos, todas las variables morfométricas y de crecimiento se correlacionaron positiva y significativamente $(\mathrm{p}<0.05)$ con el índice de calidad de Dickson (Cuadro 3). En este sentido, los resultados se aproximan a los señalados por Binotto et al. (2010) y Lima et al. (2013) quienes informaron que algunas variables de crecimiento se correlacionan fuertemente con el índice de calidad de Dickson. En el mismo contexto, Lin et al. (2019) concluyeron que las variables morfofisiológicas se corresponden con el vigor de las plántulas.

Cuadro 3. Relación entre el crecimiento y calidad de plántulas de plátano a los 60 días de aclimatación en vivero.

\begin{tabular}{lll}
\hline & \multicolumn{2}{c}{$\begin{array}{c}\text { Índice de calidad de } \\
\text { Dickson }(\mathrm{ICD})\end{array}$} \\
\hline Altura de planta $(\mathrm{cm})$ & $\mathrm{R}=0,90$ & $\mathrm{P}=<0,0001$ \\
Diámetro de tallo $(\mathrm{mm})$ & $\mathrm{R}=0,91$ & $\mathrm{P}=<0,0001$ \\
Longitud de biomasa radical $(\mathrm{cm})$ & $\mathrm{R}=0,89$ & $\mathrm{P}=<0,0001$ \\
Masa seca de planta $(\mathrm{g})$ & $\mathrm{R}=0,79$ & $\mathrm{P}=<0,0001$ \\
Área foliar $\left(\mathrm{cm}^{2}\right)$ & $\mathrm{R}=0,91$ & $\mathrm{P}=<0,0001$ \\
\hline
\end{tabular}

$\mathrm{R}=$ Coeficiente de correlación de Pearson

$\mathrm{P}=$ Significancia estadística de las correlaciones $\alpha \leq 0.05$

De acuerdo con los resultados logrados, la calidad de una plántula está en función de un desarrollo equilibrado entre las principales estructuras morfo-anatómicas y las funciones fisiológicas que cada una desempeña, lo que ha sido sugerido por varios estudios realizados con otras especies de interés agrícola (Binotto et al., 2010; Lima et al., 2013; Gomes y Miglioranza, 2015; Rezende et al., 2016; Alves et al., 2018; Lin et al., 2019). Finalmente, la calidad de una planta se traduce en mayor desarrollo del sistema radical que promueve una mayor exploración y captación de recursos nutricionales del suelo, mayor superficie foliar $\mathrm{y}$ actividad fotosintética, $\mathrm{y}$ por consiguiente mayor crecimiento y adaptación en campo.

\section{CONCLUSIONES}

La aplicación de bioestimulante en vivero fue efectivo para incrementar el crecimiento y calidad de plántulas de plátano en vivero. El bioest

imulante potenció el efecto de la fertilización edáfica. La calidad de las plántulas se correspondió con mejores características morfométricas y mayor crecimiento en vivero.

\section{AGRADECIMIENTO}

Los autores agradecemos a la Escuela Superior Politécnica Agropecuaria de Manabí Manuel Félix López, por haber financiado la totalidad de esta investigación a través de los proyectos institucionales "Selección de clones élites de plátano (Musa AAB Simmonds.) de alto potencial productivo a partir de fincas plataneras de Manabî" con CUP 381232 y "Fortalecimiento de la agricultura familiar local mediante aplicación de buenas prácticas de manejo de suelo, cultivos, bioinsumos y poscosecha" con CUP 384767 que se ejecutan actualmente en la institución, tal como consta en la Secretaría de Planificación del Estado Ecuatoriano.

\section{LITERATURA CITADA}

Ali, O., Ramsubhag, A. \& Jayaraman, J. 2021. Biostimulant Properties of Seaweed Extracts in Plants: Implications towards Sustainable Crop Production. $\quad$ Plants, 531. doi.org/10.3390/plants10030531.

Alves, R., Gomes, G., Malta, E., Andrade, G., \& do Sacramento, C. (2018). Manejo de matrizes interfere no rendimento e na qualidade de mudas de cacaueiros. Revista Brasileira de Fruticultura, 40(3): $1-8$. doi.org/10.1590/0100-29452018582.

Binotto, A., Lúcio, A. \& Lopes, S. (2010). Correlations between growth variables and the Dickson quality index in forest seedlings. Cerne, Lavras, 16(4), 457464. doi.org/10.1590/S0104-77602010000400005.

Cedeño, G., Guzmán, A., Zambrano, H., Vera, L., Valdivieso, C. \& López, G. 2020. Efecto de la densidad de siembra y riego complementario en la 
morfo-fenología, rendimiento, rentabilidad y eficiencia de la fertilización del plátano. Scientia Agropecuaria 11(4): $483-492 . \quad$ DOI: 10.17268/sci.agropecu.2020.04.03.

Colla, G., Nardi, S., Cardarelli, M., Ertani, A., Lucini, L., Canaguier, R. \& Rouphael, Y. 2015. Protein hydrolysates as biostimulants in horticulture. Scientia Horticulturae 196: 28-38. Doi.10.1016/j.scienta.2015.08.037.

Coyne, D., Wasukira, A., Dusabe, J., Rotifa, I. \& Dubois, T. 2010. Boiling water treatment: A simple, rapid and effective technique for nematode and banana weevil management in banana and plantain (Musa spp.) planting material. Crop Protection, 29, 1478-1482. doi:10.1016/j.cropro.2010.08.008.

Ewane, C., Ndongo, F., Ngoula, K., Tene, P., Opiyo, S. \& Boudjeko, T. 2019. Potential Biostimulant Effect of Clam Shells on Growth Promotion of Plantain PIF Seedlings (var. Big Ebanga \& Batard) and Relation to Black Sigatoka Disease Susceptibility. American Journal of Plant Sciences, 10: 1763-1788. DOI: 10.4236/ajps.2019.1010125.

El-Mahrouk, M. \& El-Shereif, A. 2019. Micropropagation of Banana: Reversion, Rooting, and Acclimatization of Hyperhydric Shoots. HORTSCIENCE 54(8):1384-1390. doi.org/10.21273/HORTSCI14036-19.

Falcomer, A., Resende, R., de Lima, B., Ginani, V. \& Zandonadi, R. 2019. Health Benefits of Green Banana Consumption: A Systematic Review. Nutrients, 11(6): 1222. doi: 10.3390/nu11061222.

Galán, V., Rangel, A., López, J., Pérez, J., Sandoval, J. \& Souza, H. 2018. Propagación del banano: técnicas tradicionales, nuevas tecnologías e innovaciones. Revista Brasileira de Fruticultura 40(4): 1-22. DOI: http://dx.doi.org /10.1590/0100-29452018574.

Gomes, C. \& Miglioranza, E. 2015. Quality levels of organic coffee seedlings in black and white nonwoven fabric (WNF) containers of various sizes. African Journal of Agricultural Research, 10(9), 86-89. doi.org/10.5897/AJAR2014.9393.

Grossnickle, S. \& MacDonald, E. 2018. Seedling quality: history, application, and plant attributes. A Review. Forests, 9, 283. doi:10.3390/f9050283.

INEC (Instituto Nacional de Estadísticas y Censos). 2020. Módulo de Información Agroambiental y Tecnificación Agropecuaria. Boletín técnico N-022020-MOD_AMB_ESPAC. Quito, Ecuador. 11 p.
Izquierdo, H., González, M., Núñez, M., Proenza, R. \& Álvarez, I. 2012. Efectos de la aplicación de un análogo espirostánico de brasinoesteroides en vitroplantas de banano (Musa spp.) durante la fase de aclimatización. Cultivos Tropicales, 33(1): 71-76.

Jacobsen, K., Omondi, B., Almekinders, C., Alvarez, E., Blomme, G., Dita, M., skra-Caruana, M., Ocimati, W., Tinzaara, W., Kumar, P. \& Staver, C. 2019. Seed degeneration of banana planting materials: strategies for improved farmer access to healthy seed. Plant Pathology, 68, 207-228. doi: 10.1111/ppa.12958.

Kumar, N., Krishnamoorthy, V., Nalia, L. \& Soorianathasundharam, K. 2002. A new factor for estimating total leaf area in banana. InfoMusa, 11(2), $42 \quad-\quad 43 . \quad$ Recuperado de https://www.musalit.org/seeMore.php?id=14204

Lima, S., Marimon, B., Petter, F., Tamiozzo, S., Bossi, G. \& Schwantes, B. 2013. Biochar as substitute for organic matter in the composition of substrates for seedlings. Acta Scientiarum. Agronomy, 35(3), 333341. doi. 10.4025/actasciagron.v35i3.17542.

Lin, K., Wu, C. \& Chang, Y. 2019. Applying Dickson Quality Index, Chlorophyll Fluorescence, and Leaf Area Index for Assessing Plant Quality of Pentas lanceolate. Not Bot Horti Agrobo, 47(1), 169-176. doi.10.15835/nbha47111312.

Malik, A., Mor, V., Tokas, J., Punia, H., Malik, S., Malik, K., Sangwan, S., Tomar, S., Singh, P., Singh, N., Himangini., Vikram., Nidhi., Singh, G., Vikram., Kumar, V., Sandhya. \& Karwasra, A. 2021. Biostimulant-Treated Seedlings under Sustainable Agriculture: A Global Perspective Facing Climate Change. Agronomy, 11, 14. doi.org/10.3390/agronomy11010014.

Martínez, G., Rey, J., Pargas, R., Guerra, C., Manzanilla, E. \& Ramírez, H. 2021. Efecto de sustratos y fuentes orgánicas en la propagación de banano y plátano. Agronomía Mesoamericana 32(3):808-822. doi:10.15517/am.v32i3.42490.

Mateus, D. \& Rodríguez, G. 2019. Efecto de bioestimulantes sobre la acumulación de materia seca e intercambio de gases en plantas de plátano (Musa AAB). Revista Colombiana de Ciencias Hortícolas, 13(2): 151-160. doi.org/10.17584/rcch.2019v13i2.8460.

Mora, A., Naranjo, J., Albiño, A., Flores, J., Oviedo, R., Galarza, L., Vera, M., Painii, P. \& Barcos, M. 2021. Optimización en la aclimatación de plántulas micropropagadas de banano (Musa sp.) utilizando tres 
insumos orgánicos. Bionatura 6(1):1452-1461. http://www.revistabionatura.com

Moya, M., Sánchez, E., Cabezas, D., Calderín, A., Marrero, D., Héctor, H. \& Pérez, S. 2016. Influence of vermicompost humic acid on chlorophyll content and acclimatization in banana clone, Enano Guantanamero. African Journal of Biotechnology 15(47): 2659-2670. DOI: 10.5897/AJB2016.15681.

Murray, D. 1960. The effect of deficiencies of the major nutrients on growth and leaf analysis of the banana. Tropical Agriculture, 37(2): 97-106. Recuperado de https://journals.sta.uwi.edu/ta/index.asp?action=view $\underline{\text { Issue } \& \text { issueId }=358}$

Mukhongo, R., Kavoo-Mwangi, M., Okalebo, J., Were, B., Mwangi, E. \& JJefwa, J. 2015. Acclimatization and growth of tissue cultured banana co-inoculated with microbiological and chemical commercial products in different soils in Kenya. Academia Journal of Agricultural Research 3(8): 156-168. DOI: 10.15413/ajar.2015.0113.

Parkhe, S., Dahale, M., Tayde, S. \& Nagre, P. 2019. Acclimatization of In Vitro Propagated Grand Naine Banana Plantlets. Bull. Env. Pharmacol. Life Sci., 8 (3): 44-47.

Qin, K. \& Leskovar, D. 2020. Humic Substances Improve Vegetable Seedling Quality and Post-Transplant Yield Performance under Stress Conditions. Agriculture, $\quad 10, \quad 254$. doi:10.3390/agriculture10070254.

Reyes, J., Ramos, R., Llerena, L., Ramírez, M. \& Falcón, A. 2021. Potencialidades de oligogalacturónidos y quitosacáridos en el enraizamiento de las plantas. Terra Latinoamericana 39: 1-9. e846. doi.org/10.28940/terra.v39i0.846.

Rezende, F., Hardt, V., Branco, C. \& Moura, M. 2016. Biochar in substrate composition for production of teak seedlings. Pesquisa Agropecuaria Brasileira, 51(9), 1449-1456. doi. 10.1590/S0100$204 \times 2016000900043$.
Rodríguez, G. \& Ramírez, H. 2006. Efecto de diferentes sustratos y dosis de nitrógeno sobre el desarrollo de plantas de banano (Musa AAA) en etapa de vivero. En: Soprano, E., Adami, F., Lichtemberg, L., \& Silva, M. (Editores), XVII REUNIÃO INTERNACIONAL ACORBAT 2006, Bananicultura: um negócio sustentável, Joinville - Santa Catarina - BRASIL.

Rouphael, Y. \& Colla, G. 2020. Editorial: Biostimulants in Agriculture. Front. Plant Sci. 11:40. doi: 10.3389/fpls.2020.00040.

Sepúlveda, W., Ureta, I., Hernández, G. \& Solórzano, G. 2017. Consumo de plátano en Ecuador: hábitos de compra y disponibilidad a pagar de los consumidores. Revista em Agronegócio e Meio Ambiente, Maringá (PR), 10(4): 995-1014. doi:http://dx.doi.org/10.17765/21769168.2017v10n4p995-1014.

Scott, G. 2021. A review of root, tuber and banana crops in developing countries: past, present and future. International Journal of Food Science and Technology, 56: 1093-1114. doi:10.1111/ijfs.14778.

Staver, C. \& Lescot, T. 2015. La propagación de material de siembra de calidad para mejorar la salud y productividad del cultivo: prácticas clave para las musaceas: Guía ilustrada. Bioversity International, Roma, IT. 26 p. ISBN 13:978-92-9255-016-5. Recuperado de https://www.bioversityinternational.org/fileadmin/us er upload/online library/publications/pdfs/multiplic ation_guide_Spanish_bookmarked_opt.pdf

Zambrano, Y., Rivadeneira, J. \& Pérez, M. 2018. Linking El Niño Southern Oscillation for early drought detection in tropical climates: The Ecuadorian coast. Science of the Total Environment 643: 193-207. doi.10.1016/j.scitotenv.2018.06.160. 